\title{
Shock acceleration as origin of the radio relic in A 521?
}

\author{
S. Giacintucci ${ }^{1,2}$, T. Venturi ${ }^{1}$, G. Macario ${ }^{1,3}$, D. Dallacasa ${ }^{1,3}$, G. Brunetti ${ }^{1}$, M. Markevitch ${ }^{2}$, \\ R. Cassano ${ }^{1,3}$, S. Bardelli ${ }^{4}$, and R. Athreya ${ }^{5}$ \\ 1 INAF - Istituto di Radioastronomia, Via Gobetti 101, 40129 Bologna, Italy \\ e-mail: sgiaci_s@ira.inaf.it \\ 2 Harvard-Smithsonian Centre for Astrophysics, 60 Garden Street, Cambridge, MA 02138, USA \\ 3 Dipartimento di Astronomia, Università di Bologna, via Ranzani 1, 40126 Bologna, Italy \\ ${ }^{4}$ INAF - Osservatorio Astronomico di Bologna, via Ranzani 1, 40126 Bologna, Italy \\ 5 Tata Institute of Fundamental Research, National Centre for Radio Astrophysics, Ganeshkhind, Pune 411007, India
}

Received 25 January 2008 / Accepted 22 March 2008

\section{ABSTRACT}

\begin{abstract}
Aims. We present new high sensitivity observations of the radio relic in A 521 carried out using the Giant Metrewave Radio Telescope at $327 \mathrm{MHz}$ and with the Very Large Array at 4.9 and $8.5 \mathrm{GHz}$.

Methods. We imaged the relic at these frequencies and carried out a detailed spectral analysis, based on the integrated radio spectrum between $235 \mathrm{MHz}$ and $4.9 \mathrm{GHz}$, and on the spectral index image in the frequency range 327-610 MHz. In our present analysis we use our new GMRT observations in addition to proprietary and archival data. We search for a possible shock front co-located with the relic on a short archival Chandra X-ray observation of the cluster.

Results. The integrated spectrum of the relic is consistent with a single power law; the spectral index image shows a clear trend of steepening going from the outer portion of the relic toward the cluster centre. We discuss the origin of the source in the light of theoretical models for the formation of cluster radio relics. Our results on the spectral properties of the relic are consistent with acceleration of relativistic electrons by a shock in the intracluster medium. This scenario is supported by our detection of an X-ray surface brightness edge coincident with the outer border of the radio relic. This edge is probably a shock front.
\end{abstract}

Key words. radio continuum: galaxies - galaxies: clusters: general - galaxies: clusters: individual: A 521

\section{Cluster radio relics and the case of $\mathbf{A} 521$}

The radio emission from clusters of galaxies comes into two flavours. In addition to the individual radio sources associated with cluster galaxies, a fraction of massive and X-ray luminous clusters with clear signature of ongoing mergers, hosts large scale diffuse radio sources, known as radio halos, if located at the cluster centre, and relics, if located in peripheral regions. The synchrotron emission in these diffuse sources arises directly within the intracluster medium (ICM), and probes the existence of non-thermal components spread over the cluster scale (e.g., the review paper by Feretti 2003).

Particle acceleration by means of turbulence injected into the cluster volume during mergers represents a promising possibility to understand the origin of radio halos (see the review papers by Brunetti 2003, 2004; Sarazin 2004; Petrosian \& Bykov 2008). Statistical analyses (e.g., Kuo et al. 2004; Cassano \& Brunetti 2005; Cassano et al. 2006, 2008) and deep radio observations of samples of galaxy clusters (Brunetti et al. 2007; Venturi et al. 2007, 2008) have provided further support to this scenario. On the other hand, our understanding of the origin of relics is still limited. Only a handful of radio relics have been observed in some detail (e.g., A 2256, Clarke \& Enßlin 2006; A 3667, Röttgering et al. 1997; A 2744, Orrú et al. 2007). Integrated radio spectra over a wide range of frequencies are available for a few relics only (e.g., 1257+275 in the Coma cluster; Andernach et al. 1984; Thierbach et al. 2003, and references therein). All models proposed so far for the relic formation invoke the presence of a shock within the X-ray gas (Enßlin et al. 1998; Roettiger et al. 1999; Enßlin \& Gopal-Krishna 2001; Hoeft \& Brüggen 2007).

We focus our attention on the radio relic in A 521 (Ferrari et al. 2006; Giacintucci et al. 2006, hereinafter GVB06), an $\mathrm{X}$-ray luminous and massive galaxy cluster $\left(L_{\mathrm{X}}[0.1-2.4 \mathrm{keV}] \sim\right.$ $8 \times 10^{44} \mathrm{erg} \mathrm{s}^{-1}$; virial mass $M_{\mathrm{v}} \sim 1.9 \times 10^{15} M_{\odot}^{1}$ ) at redshift $z=0.247$. Multiple merging episodes are known to occur in this disturbed cluster whose properties are indicative of an object in a complex dynamical state, which is still accreting a number of smaller mass concentrations (e.g., Maurogordato et al. 2000; Ferrari et al. 2003, 2006; see also Fig. 1 in GVB06 for a sketch of the multiple optical and X-ray substructures in the cluster).

A radio study of A 521 based on $610 \mathrm{MHz}$ Giant Metrewave Radio Telescope (GMRT) observations (GVB06) shows that the relic is a diffuse elongated structure located in the southeastern periphery of A 521, on the edge of a dynamically active region where galaxy groups are infalling into the main cluster (Maurogordato et al. 2000; Ferrari et al. 2003). The relic is at the boundary of the X-ray emission from the intracluster gas, at a projected distance of $\sim 930 \mathrm{kpc}$ from the cluster X-ray centre, and is apparently connected to the most powerful radio galaxy in A 521 (J0454-1016a) by a faint bridge of radio emission. Even though projection effects should be taken into account, this situation is similar to what is observed in the Coma cluster, where a bridge of radio emission connects the tails of the radio galaxy

\footnotetext{
1 From the correlation between the X-ray luminosity and $M_{\mathrm{v}}$ reported in Cassano et al. (2006).
} 
Table 1. Summary of the radio observations.

\begin{tabular}{lcccccccc}
\hline \hline Telescope & $\begin{array}{c}\mathrm{RA}_{\mathrm{J} 2000} \\
(\mathrm{~h}, \mathrm{~m}, \mathrm{~s})\end{array}$ & $\begin{array}{c}\mathrm{Dec}_{\mathrm{J} 2000} \\
\left({ }^{\circ},{ }^{\prime},{ }^{\prime}\right)\end{array}$ & $\begin{array}{c}\text { Observation } \\
\text { date }\end{array}$ & $\begin{array}{c}v \\
\mathrm{MHz}\end{array}$ & $\begin{array}{c}\Delta v \\
\mathrm{MHz}\end{array}$ & $\begin{array}{c}t \\
\mathrm{~min}\end{array}$ & $\begin{array}{c}H P B W, \mathrm{PA} \\
\left(\text { full array, }, \times ”,{ }^{\circ}\right)\end{array}$ & $\begin{array}{c}\mathrm{rms} \\
\mu \mathrm{Jy} \mathrm{b} \mathrm{b}^{-1}\end{array}$ \\
\hline GMRT & 045409.00 & -101419.0 & Nov. 06 & 327 & $16 \diamond$ & 330 & $10.6 \times 9.6,-19$ & $90-100 *$ \\
VLA-BnA & 045416.28 & -101605.9 & Jun. 06 & 4860 & 50 & 110 & $1.0 \times 0.8,-68$ & 10 \\
VLA-CnB & 045416.28 & -101605.9 & Oct. 06 & 4860 & 50 & 60 & $4.0 \times 2.0,-82$ & 15 \\
VLA-BnA & 045416.28 & -101605.9 & Jun. 06 & 8430 & 50 & 170 & $0.6 \times 0.5,82$ & 8 \\
\hline
\end{tabular}

$\diamond$ The observations were performed using a total bandwidth of $32 \mathrm{MHz}$ (USB+LSB), but only the USB dataset was used for the analysis (see Sect. 2.1).

* The rms noise level in the region of the relic is $\sim 90 \mu \mathrm{Jy} \mathrm{b}^{-1}$, and increases up to $\sim 100 \mu \mathrm{Jy} \mathrm{b}^{-1}$ in the outer parts of the A 521 field due to the presence of strong radio sources.

NGC 4789 to the prototype relic source $1253+275$ (Giovannini et al. 1991).

We present a study of the origin of the relic based on new GMRT $327 \mathrm{MHz}$ high sensitivity observations of A 521 and new high frequency and high resolution images of the relic region obtained from Very Large Array (VLA) observations at 4.9 and 8.5 GHz. The amount of radio information available allows us to study in detail the spectral properties of the source (integrated and point-to-point) over a relatively wide range of frequencies, and compare them to the model predictions of its origin. The paper is organised as follows: Sect. 2 describes the radio observations and data reduction; the new GMRT $327 \mathrm{MHz}$ images of the A 521 field and relic region are presented in Sect. 3; in Sect. 4, we report on the analysis of the VLA 4.9 and $8.5 \mathrm{GHz}$ images; Sect. 5 deals with the study of the spectral index image; the source integrated radio spectrum is analysed in Sect. 6; the proposed scenarios for the relic origin are discussed in Sect. 7; finally a summary of our results is given in Sect. 8 .

We adopt the $\Lambda \mathrm{CDM}$ cosmology with $H_{0}=$ $70 \mathrm{~km} \mathrm{~s}^{-1} \mathrm{Mpc}^{-1}, \Omega_{\mathrm{m}}=0.3$ and $\Omega_{\Lambda}=0.7$. At the redshift of A 521, this cosmology leads to a linear scale of $1^{\prime \prime}=3.87 \mathrm{kpc}$. The spectral index $\alpha$ is defined according to $S \propto v^{-\alpha}$.

\section{Radio observations and data reduction}

We carried out high sensitivity observations of A 521 using the GMRT at $327 \mathrm{MHz}$. To image the sky region close to the relic at higher frequency and resolution, and resolve the inner structure of the radio galaxy J0454-1016a, we performed VLA observations at $4.9 \mathrm{GHz}$ in the hybrid $\mathrm{BnA}$ and $\mathrm{CnB}$ configurations, and at $8.5 \mathrm{GHz}$ in the BnA configuration. In Table 1, we summarise the details of all the radio observations presented in this paper. Columns in the table provide the following information: telescope/array; J2000 coordinates of the pointing centre; observing day; frequency; total bandwidth; total time on source; half power bandwidth $(H P B W)$ and rms level $(1 \sigma)$ in the full resolution image.

\subsection{GMRT observations at $327 \mathrm{MHz}$}

A 521 was observed using the GMRT at $327 \mathrm{MHz}$ in November 2006 for a total integration time of $5.5 \mathrm{~h}$ (Table 1). These observations were performed using both the upper and lower side band (USB and LSB, respectively) for a total observing bandwidth of $32 \mathrm{MHz}$. The data were collected in spectralline mode with 128 channels/band, and a spectral resolution of $125 \mathrm{kHz} /$ channel.
The USB and LSB datasets were calibrated and analysed individually using the NRAO Astronomical Image Processing System (AIPS) package. The bandpass calibration was performed using the flux density calibrator. A RFI-free channel was chosen to normalise the bandpass for each antenna. The calibration solutions were applied to the data by running the AIPS task FLGIT, which subtracts a continuum from the channels in the $u-v$ plane, determined on the basis of the bandpass shape and using a specified set of channels. The data whose residuals exceed a chosen threshold are then flagged. Despite this flagging procedure, both the USB and LSB datasets were still affected by strong residual radio frequency interferences (RFI). Hence, an accurate editing of the visibility data was carried out to identify and remove data affected by RFI.

To find a compromise between the size of the dataset and the need to minimize bandwidth smearing effects within the primary beam, the central channels were averaged to 6 channels of $\sim 2 \mathrm{MHz}$ each after bandpass calibration. Given the large field of view of the GMRT, in each step of the data reduction we implemented the wide-field imaging technique to minimise the errors due to the non-planar nature of the sky. We used 25 facets covering a total field of view of $\sim 2.7 \times 2.7$ square degrees. After a number of phase self-calibration cycles, the final USB and LSB datasets were averaged further from 6 channels to 1 single channel $^{2}$.

Due to residual phase errors in the LSB dataset, the USBLSB data combination led to images with a quality worse than those obtained from the USB alone. For this reason, only the USB dataset was used for the analysis presented in this present paper. A very high sensitivity $(1 \sigma)$ was achieved in our final full resolution image (Table 1): from $\sim 90 \mu \mathrm{Jy} \mathrm{b}^{-1}$ in the region of the relic to $\sim 100 \mu \mathrm{Jy} \mathrm{b}^{-1}$ in the outer parts of the A 521 field, where the quality of the image is limited by the presence of strong radio sources. The residual amplitude errors are approximately $\lesssim 5 \%$.

\subsection{VLA observations at 4.9 and $8.5 \mathrm{GHz}$}

The $4.9 \mathrm{GHz}$ observations of the relic region were carried out using the VLA in the hybrid BnA and $\mathrm{CnB}$ configurations. The 8.5 GHz observations were performed in the BnA configuration (see Table 1 for details). A bandwidth of $50 \mathrm{MHz}$ was used for each of the two IF channels at each frequency. All observations included full polarisation information. The data were calibrated and reduced using the pilot semi-automatic pipeline for the VLA data processing, a facility developed at NRAO and implemented in the AIPS package. The pipeline provided high quality

\footnotetext{
2 Bandwidth smearing is relevant only on the outskirts of the wide field, and does not significantly affect the region presented and analysed in this paper.
} 


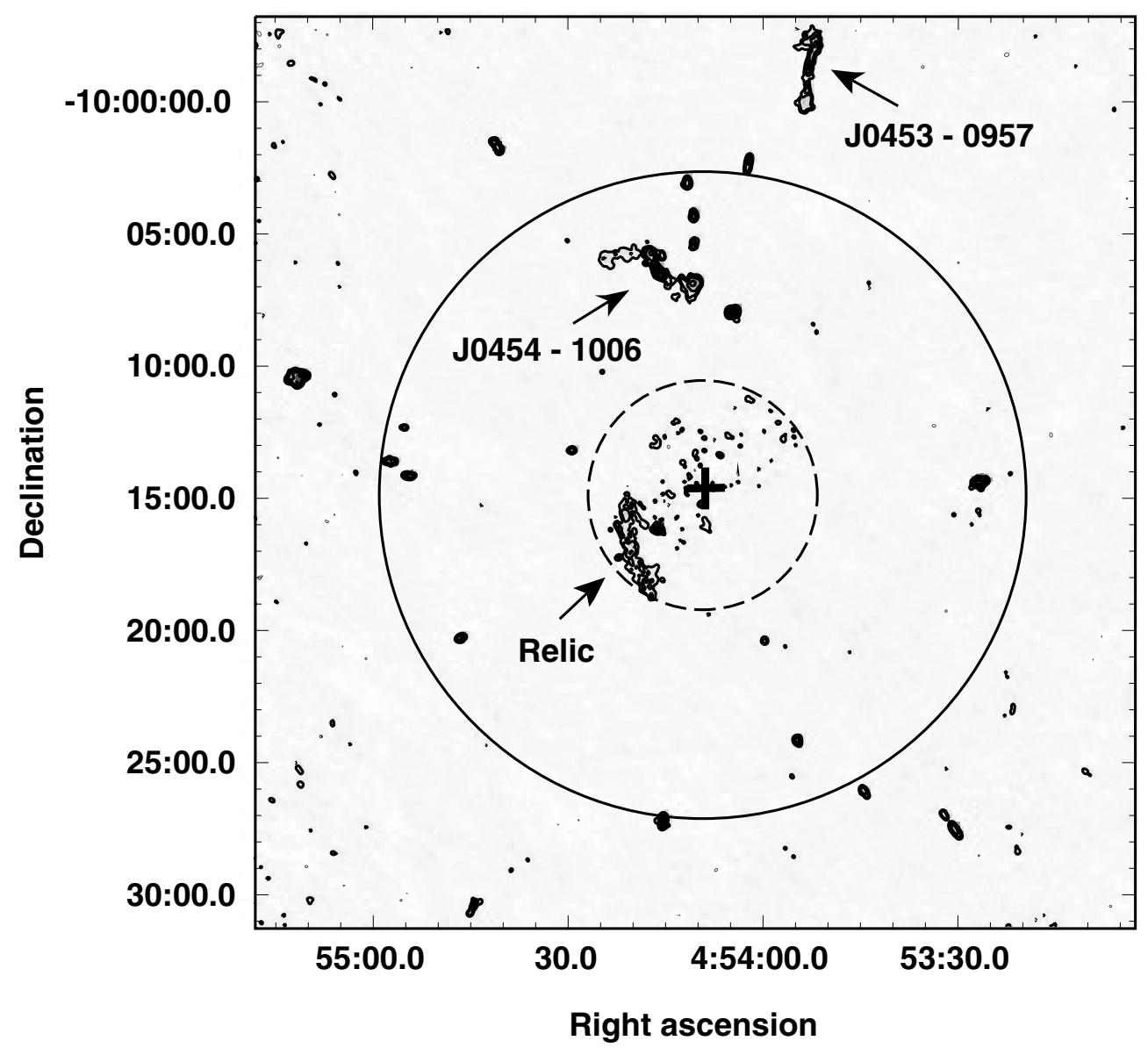

Fig. 1. GMRT $327 \mathrm{MHz}$ contours of the $30^{\prime} \times 30^{\prime}$ region containing A 521 . Contour levels are spaced by a factor 2 starting from $5 \sigma= \pm 0.5 \mathrm{mJy} \mathrm{b}^{-1}$. The resolution is $10.6^{\prime \prime} \times 9.6^{\prime \prime}$, in PA $-19^{\circ}$. The cross marks the X-ray centre of the cluster (Arnaud et al. 2000). The radius of the solid circle corresponds to the cluster virial radius $R_{\mathrm{v}}=2.8 \mathrm{Mpc}(\mathrm{GVB} 06)$. The dashed circle has a radius of $\sim 1 \mathrm{Mpc}$, and indicates the region covered by the optical analysis in Ferrari et al. (2003).

calibrated datasets at each frequency. These datasets were further phase self-calibrated in AIPS, to correct for residual phase variations, and used to produce the final images. The rms noise level achieved in the final images are reported in Table 1 . The average residual amplitude errors in the data are approximately $\lesssim 5 \%$ both at 4.9 and $8.5 \mathrm{GHz}$.

\section{A 521 at $327 \mathrm{MHz}$}

\subsection{The field}

In Fig. 1, we present the GMRT full resolution image at $327 \mathrm{MHz}$ that covers the region within the cluster virial radius $\left(R_{\mathrm{V}}=2.78 \mathrm{Mpc}\right.$; GVB06), which is delimited by the solid circle. The figure shows the same $\sim 30^{\prime} \times 30^{\prime}$ field presented at $610 \mathrm{MHz}$ in Fig. 2 of GVB06. The cross marks the centre of the cluster X-ray emission as detected by ROSAT HRI (Arnaud et al. 2000). The dashed circle, which has a radius corresponding to $\sim 1 \mathrm{Mpc}$, indicates the area covered by the analysis of the optical substructures in Ferrari et al. (2003). The radio contours are plotted starting from $\pm 0.5 \mathrm{mJy} \mathrm{b}^{-1}$, which corresponds to the $5 \sigma$ detection significance level in the region with the highest noise.

Three extended radio sources are visible in the image: the radio relic, in the southeastern outskirts of the cluster, and the two radio galaxies J0453-0957 and J0454-1006, located North of A 521, and analysed at $610 \mathrm{MHz}$ in GVB06.
In addition to discrete point sources, positive residuals of radio emission are detected within the dashed circle in Fig. 1, which suggest that diffuse emission is present at the cluster centre. The investigation of this point is beyond the purpose of the present paper, and will be addressed in a forthcoming paper (Brunetti et al., to be submitted).

\subsection{The radio relic at $327 \mathrm{MHz}$}

As observed at higher frequencies, the $327 \mathrm{MHz}$ radio emission within the inner $\sim 1 \mathrm{Mpc}$ radius is clearly dominated by the relic. Figure 2 zooms into the $327 \mathrm{MHz}$ image of the relic. In the left panel, we show the full resolution contours overlaid on the optical POSS-2 frame (grey scale). Labels A, B and C indicate the position of the radio galaxies embedded in the diffuse relic emission, and optically identified in GVB06. In the right panel, we show an image at the resolution of $15.0^{\prime \prime} \times 12.0^{\prime \prime}$ with grey scale and contours overlaid to highlight the distribution of the radio surface brightness across the source. The relic exhibits a highly elongated and arc-shaped structure with an angular size of $\sim 4.3^{\prime}$, which corresponds to a linear size of $\sim 1 \mathrm{Mpc}$. The overall morphology and total extent in Fig. 2 are in good agreement with the images at $610 \mathrm{MHz}$ (GVB06; also reported in Fig. 5) and at $1.4 \mathrm{GHz}$ (Ferrari et al. 2006) of similar resolution. The relic emission along the minor axis appears, on average, to be slightly wider in the $327 \mathrm{MHz}$ image $\left(\sim 1.0^{\prime}\right.$, i.e., $\left.\sim 230 \mathrm{kpc}\right)$ 

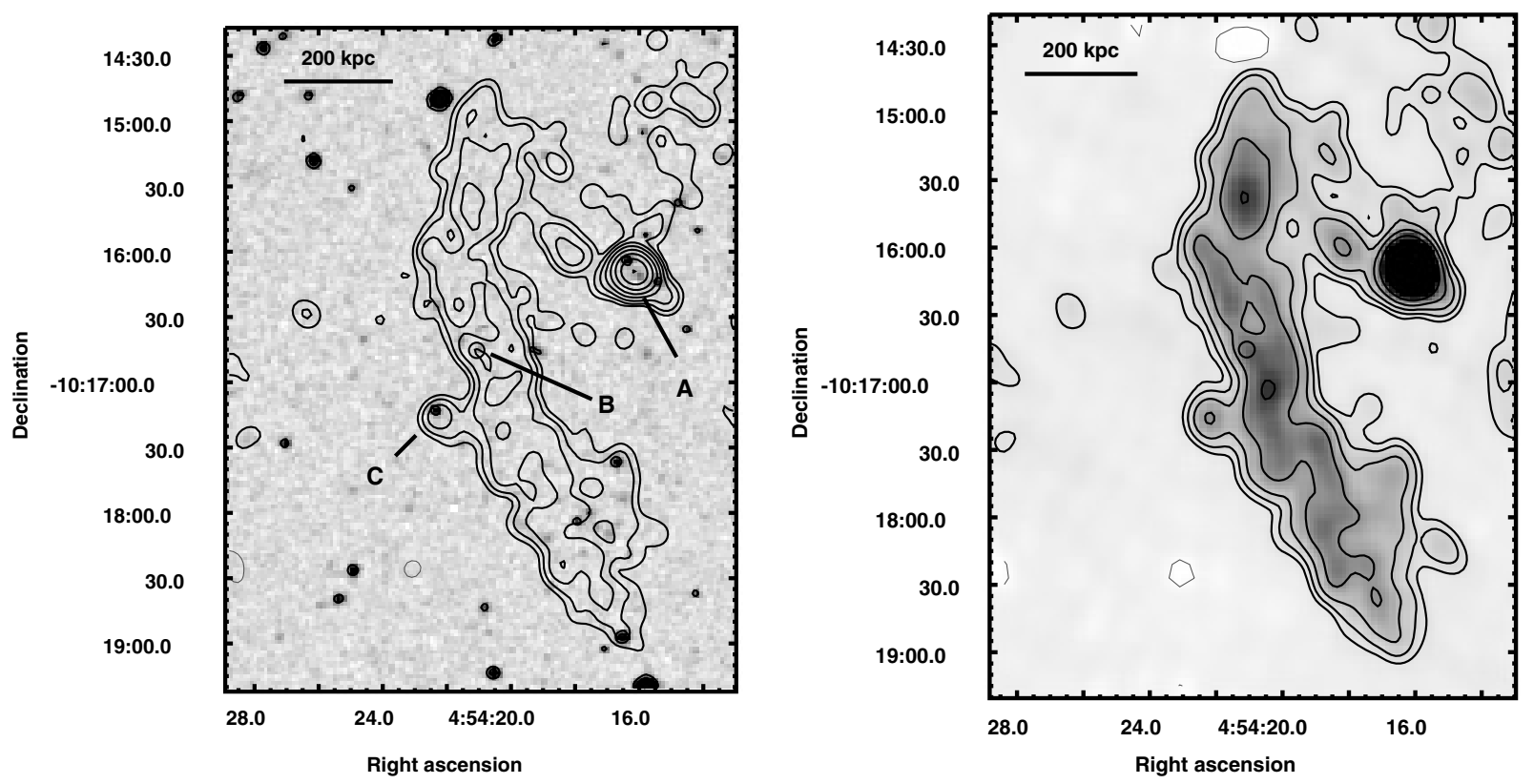

Fig. 2. Left panel: full resolution GMRT contours at $327 \mathrm{MHz}$ of the relic, overlaid on the red POSS-2 optical image. The resolution is $10.6^{\prime \prime} \times 9.6^{\prime \prime}$, in PA $-19^{\circ}$. Contours are spaced by a factor 2 starting from $\pm 3 \sigma=0.27 \mathrm{mJy} \mathrm{b}^{-1}$. A, B and C indicate the position of the radio galaxies embedded in the relic emission. Right panel: low resolution GMRT image at $327 \mathrm{MHz}$ of the relic (contours and grey scale). The resolution is $15.0^{\prime \prime} \times 12.0^{\prime \prime}$, in PA $0^{\circ}$. Contours are spaced by a factor 2 starting from $\pm 3 \sigma=0.27 \mathrm{mJy} \mathrm{b}^{-1}$.

than at higher frequencies $(\sim 200 \mathrm{kpc}$ at $610 \mathrm{MHz}$ and $\sim 160 \mathrm{kpc}$ at $1.4 \mathrm{GHz}$ ).

\section{The relic region at 4.9 and $8.5 \mathrm{GHz}$}

\subsection{The radio galaxy J0454-1016a}

Source A in Fig. 2 is the cluster radio galaxy J0454-1016a. A faint bridge of emission is clearly detected between the northern part of the relic and this radio galaxy, which is located at a projected distance of approximately $350 \mathrm{kpc}$ from the relic. This emission was also observed at $610 \mathrm{MHz}$, and suggested the presence of a physical link between the relic and J0454-1016a (GVB06). We studied the possible connection between these sources using VLA 4.9 and $8.5 \mathrm{GHz}$ observations (Table 1), which resolve the inner structure of J0454-1016a and enable us to search for connections with the nearby relic in the form, for example, of bent jets and/or extended emission in that direction.

J0454-1016a is the most powerful radio galaxy in A 521 (GVB06; Table 2), and is identified with the galaxy \#143 $\left(v=74282 \mathrm{~km} \mathrm{~s}^{-1}, I=17.00\right)$ in the optical catalogue by Ferrari et al. (2003). Figure 3 shows the region of the relic and J0454-1016a (labelled as A) in increasing frequency (from $610 \mathrm{MHz}$ to $8.5 \mathrm{GHz})$ and resolution order $\left(\sim 5^{\prime \prime}\right.$ to $\left.\sim 0.5^{\prime \prime}\right)$ going from panels $a$ ) to $d$ ). Panel $a$ ) shows the full resolution image at $610 \mathrm{MHz}$. The VLA-CnB $4.9 \mathrm{GHz}$ image of $\mathrm{J} 0454-1016 \mathrm{a}$ is presented in panel $b$ ); the BnA full resolution images at 4.9 and $8.5 \mathrm{GHz}$ are shown in panels $c$ ) and $d$ ), respectively. J0454-1016a appears extended in all images with a largest linear size of $\sim 30 \mathrm{kpc}$. Its radio structure is consistent with a head-tail morphology. The compact component, detected at 4.9 and $8.4 \mathrm{GHz}$ (panels $c$ and $d$ ), is coincident with the nucleus of the host galaxy.

The extended emission is entirely located southwest of the compact component, and there is no evidence of any emission in the direction of the radio bridge detected at $327 \mathrm{MHz}$ (Fig. 2) and $610 \mathrm{MHz}$ (panel $a$ ). This result might rule out a physical
Table 2. Properties of the radio galaxy J0454-1016a.

\begin{tabular}{lc}
\hline \hline$S_{327 \mathrm{MHz}}(\mathrm{mJy})$ & $46.0 \pm 2.3^{a}$ \\
$S_{610 \mathrm{MHz}}(\mathrm{mJy})$ & $27.7 \pm 1.4^{b}$ \\
$S_{1.4 \mathrm{GHz}}(\mathrm{mJy})$ & $15.0 \pm 0.8^{c}$ \\
$S_{4.9 \mathrm{GHz}}(\mathrm{mJy})$ & $6.2 \pm 0.3^{d}$ \\
$S_{8.5 \mathrm{GHz}}(\mathrm{mJy})$ & $3.6 \pm 0.2^{e}$ \\
$\left.\log P_{1.4 \mathrm{GHz}}(\mathrm{W} \mathrm{Hz})^{-1}\right)$ & 24.41 \\
$\alpha_{4.9 \mathrm{GHz}}^{8.5 \mathrm{GHz}}$ & $0.79 \pm 0.13$ \\
\hline
\end{tabular}

Notes to Table 2: ${ }^{a}$ from Fig. 2 (left panel); ${ }^{b}$ from GVB06; ${ }^{c}$ from VLA archival data (Obs. Id. AF 0390); ${ }^{d}$ from panel $b$ ) of Fig. $3 ;{ }^{e}$ from panel $d$ ) of Fig. 3.

connection between the diffuse emission from the relic and this radio galaxy, whose tail extends (at least in projection) in almost the opposite direction.

The flux densities available for J0454-1016a are presented in Table 2, where we also report the $1.4 \mathrm{GHz}$ radio power (from VLA archival data of program AF 0390, data which were reanalysed by Giacintucci 2007), and the spectral index in the $327 \mathrm{MHz}-8.5 \mathrm{GHz}$ interval. The flux density measurements on both images at $4.9 \mathrm{GHz}$ (panels $b$ and $c$ in Fig. 3) are consistent within the errors. Figure 4 shows the integrated radio spectrum of J0454-1016a between $327 \mathrm{MHz}$ and $8.4 \mathrm{GHz}$, derived using the values in Table 2 .

We fitted the spectrum with a a continuous injection model (CI; Kardashev 1962), using the Synage++ package (Murgia 2001). The best fit is shown as a solid line in Fig. 4, and provides an injection spectral index $\alpha_{\text {inj }}=0.70_{-0.14}^{+0.10}$. Even though there is an indication of a spectral steepening above $4.9 \mathrm{GHz}$, the spectrum is consistent with a single power law of slope $\alpha=\alpha_{\text {inj }}$.

The spectral shape in Fig. 4 is similar to that observed in other active and low luminosity radio galaxies (e.g., Parma et al. 2002). 


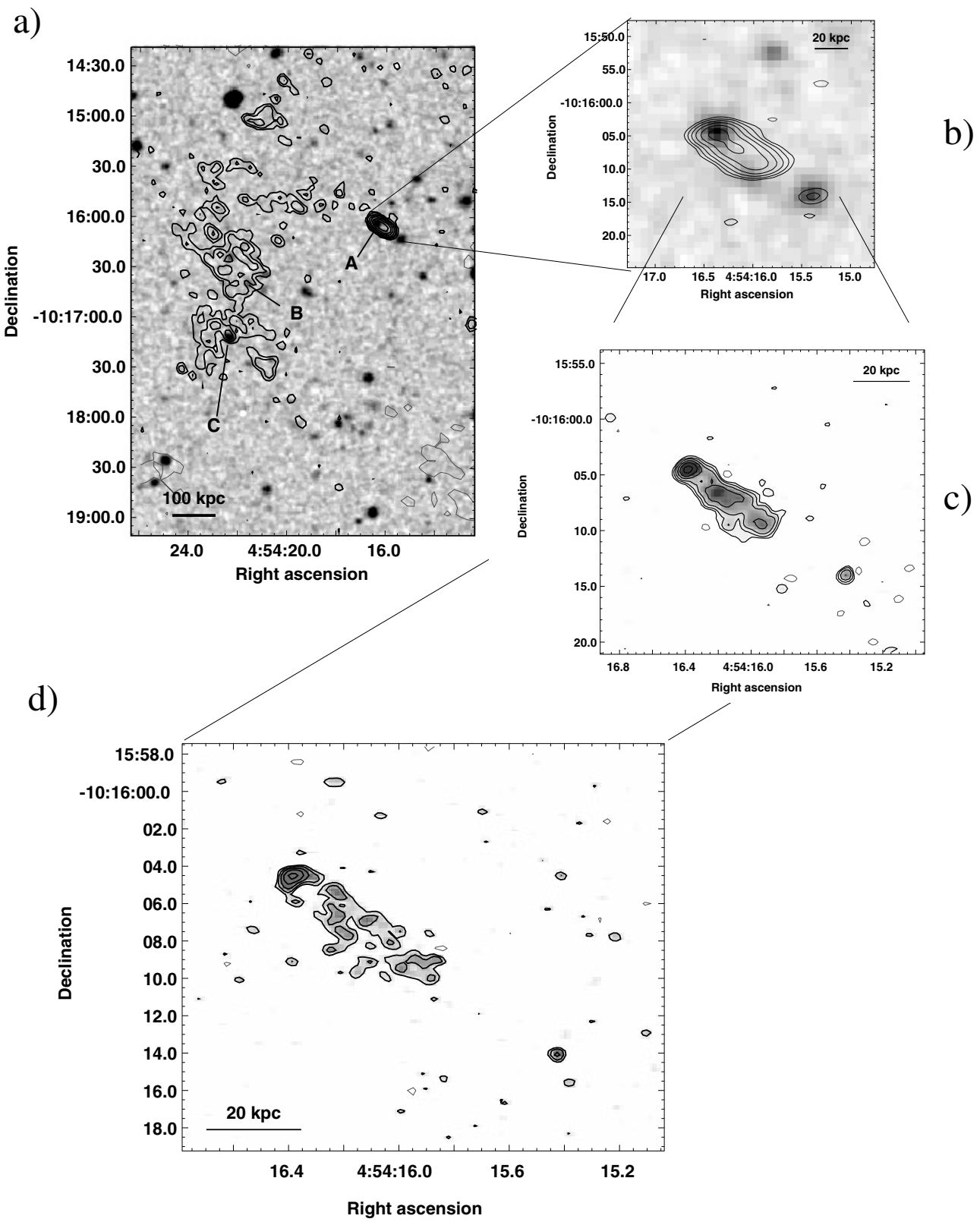

Fig. 3. Panel a) GMRT $610 \mathrm{MHz}$ full resolution contours of the relic on the POSS-2 image. The HPBW is $5.0^{\prime \prime} \times 4.0^{\prime \prime}$. The $1 \sigma$ noise level is $40 \mu \mathrm{Jy} \mathrm{b}^{-1}$. A, B and C indicate the position of radio galaxies embedded in the relic emission. Panel b) VLA-CnB 4.9 GHz contours of J0454-1016a on the POSS-2; $H P B W=4.0^{\prime \prime} \times 2.0^{\prime \prime} ; 1 \sigma=15 \mu \mathrm{Jy} \mathrm{b}^{-1}$. Panel c) VLA-BnA $4.9 \mathrm{GHz}$ image (contours and grey scale) of J0454-1016a; $H P B W=1.0^{\prime \prime} \times 0.8^{\prime \prime} ; 1 \sigma=10 \mu \mathrm{Jy} \mathrm{b}^{-1}$. Panel d) VLA-BnA $8.4 \mathrm{GHz}$ image (contours and grey scale) of J0454-1016a. HPBW $=0.6^{\prime \prime} \times 0.5^{\prime \prime}$; $1 \sigma=8 \mu \mathrm{Jy} \mathrm{b}^{-1}$. In all the panels, the contour levels are spaced by a factor 2 starting from a significance level $\pm 3 \sigma$.

\subsection{The radio relic at $4.9 \mathrm{GHz}$}

The most interesting result of the VLA $4.9 \mathrm{GHz}$ observations is the detection of the radio relic, shown in Fig. 5. This is the second detection of a radio relic at a frequency as high as $4.9 \mathrm{GHz}$, after the relic source $1253+275$ in the Coma cluster (Andernach et al. 1984; Thierbach et al. 2003). The image was obtained from the VLA-CnB data (Table 1), tapered to a resolution of $22.0^{\prime \prime} \times 15.0^{\prime \prime}$. In the left panel, the relic is shown as contours, while in the right panel the $4.9 \mathrm{GHz}$ emission is reported in grey scale with the GMRT $610 \mathrm{MHz}$ contours overlaid (GVB06). The faintest features of the relic emission are significant at the $3 \sigma$ level $\left(1 \sigma=15 \mu \mathrm{Jy} \mathrm{b}^{-1}\right)$, and the peaks at the level of $12 \sigma$. We point out that, given the short observing time (Table 1) and lack of short baselines (minimum baseline $\sim 1 \mathrm{k} \lambda$ ), the $u-v$ coverage of the $4.9 \mathrm{GHz}$ observations is inadequate to properly image the entire diffuse emission associated with the relic. The details of the structure of the source in Fig. 5 may not be therefore fully reliable, and deeper $4.9 \mathrm{GHz}$ observations using a more appropriate array and $u-v$ coverage are required to determine more accurately the relic morphology at this frequency.

\section{Spectral index image of the relic}

Spectral index imaging of cluster radio relics is a powerful tool to attempt to understand the origin, evolution, and connection of the relics with the merging activity of the host cluster. In particular, the spectral index images provide important information on the energy spectrum of the radio emitting electrons and magnetic field distribution in these sources (e.g., Clarke \& Enßlin 2006). 


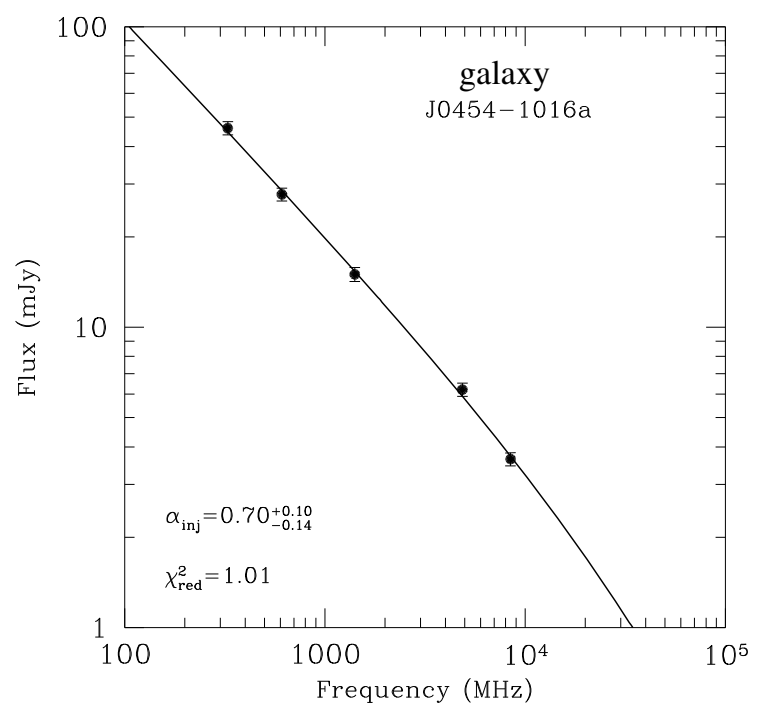

Fig. 4. Integrated radio spectrum of J0454-1016a between $327 \mathrm{MHz}$ and $8.5 \mathrm{GHz}$. The solid line is the best fit of the CI model to the data. The value of $\alpha_{\text {inj }}$ provided by the fit, along with the reduced $\chi^{2}$, is also reported.

Table 3. Details of the $327-610 \mathrm{MHz}$ spectral index image.

\begin{tabular}{lc}
\hline \hline Frequency range $\left(v_{1}-v_{2}\right)$ & $327-610 \mathrm{MHz}$ \\
$u-v$ range & $0.12-25 \mathrm{k} \lambda$ \\
$H P B W, \mathrm{PA}$ & $15.0^{\prime \prime} \times 12.0^{\prime \prime}, 0^{\circ}$ \\
$\mathrm{rms}\left(v_{1}\right)$ & $90 \mu \mathrm{Jy} \mathrm{b}^{-1}$ \\
$\mathrm{rms}\left(v_{2}\right)$ & $40 \mu \mathrm{Jy} \mathrm{b}^{-1}$ \\
\hline
\end{tabular}

We made an image of the spectral index distribution in the A 521 relic, for the frequency range $327-610 \mathrm{MHz}$, by comparing the GMRT image at $327 \mathrm{MHz}$, shown here (Fig. 2; right panel), with the GMRT $610 \mathrm{MHz}$ image obtained from the observations presented in GVB06. The images were produced using the same cell size, $u-v$ range, and restoring beam. For details, we refer to Table 3, where we provide the $u-v$ range, beam, and noise level $(1 \sigma)$ of the two images. The images were aligned, pixels with brightness below the $3 \sigma$ level were blanked, and the image combination was carried out to create the spectral index image using the Synage++ package. The resulting spectral index image is shown in Fig. 6 (colour), with the $610 \mathrm{MHz}$ contours overlaid.

\subsection{General features of the spectral index image}

The distribution of the spectral index in Fig. 6 shows different features along the two axes of the relic. Along the major axis, a number of irregularities provide a rather patchy appearance of the spectral index image. Such patchiness might arise from irregularities in the $u-v$ coverage that occur at different spacings for the two frequencies. Along the minor axis, a steepening of the spectral index from the eastern edge of the relic toward the western border is visible. This trend is real and not driven by a misalignment between the images at 327 and $610 \mathrm{MHz}$. We checked carefully that the images were aligned correctly using point sources in the relic region. The spectral index distribution appears uniform within each of the point sources and consistent with the value of $\alpha$ derived from their total flux density at the two frequencies. This is clear from Fig. 6, where, for example, the radio galaxy $\mathrm{J} 0454-1016$ a (labelled as A) has an average spectral index $\alpha=0.75 \pm 0.05$, which agrees with the source integrated spectrum shown in Fig. 4 (see also Table 2).

\subsection{Analysis of the radial steepening}

To check the significance of the spectral steepening visible in Fig. 6, we determined the average spectral index in 3 independent strips of dimensions $14^{\prime \prime} \times 80^{\prime \prime}$ in the northern region of the relic and 3 independent strips of dimensions $14^{\prime \prime} \times 100^{\prime \prime}$ in the southern part. These two regions are labelled respectively $\mathrm{N}$ and $S$ in the left panel of Fig. 7. The central portion of the relic was excluded from the analysis because of the presence of the radio galaxies $\mathrm{B}$ and $\mathrm{C}$ (Fig. 7), which may affect the spectral trend. The strips were set parallel to the edge of the relic, i.e., at a position angle of $5^{\circ}$ and $32^{\circ}$ in the $\mathrm{N}$ and $\mathrm{S}$ regions, respectively. For each strip, we integrated the flux density in the $327 \mathrm{MHz}$ and $610 \mathrm{MHz}$ images individually, and calculated the corresponding spectral index values. The average $327-610 \mathrm{MHz}$ spectral index in each strip is shown in the right panel of Fig. 7. The spectral index trend shows a gradual steepening going westwards both in the $\mathrm{N}$ and $\mathrm{S}$ regions. In particular, $\alpha$ ranges from $\simeq 1.0 \pm 0.1$ to $\simeq 2.1 \pm 0.1$ in the southern part (empty triangles), and from $\simeq 1.3 \pm 0.2$ to $\simeq 2.3 \pm 0.1$ across the northern region (filled triangles).

A steepening from the outer to the inner edge of the relic with available spectral index images has been observed in a few other cases, for example in A 3667 (Röttgering et al. 1997) and A 2744 (Orrú et al. 2007). A more detailed study of the spectral index gradient, similar to the analysis presented in this paper, was carried out for the relic in A 2256 by Clarke \& Enßlin (2006), who found a significant steepening of $\alpha$ between $1369 \mathrm{MHz}$ and $1703 \mathrm{MHz}$ from the external edge of the relic toward the cluster core, i.e., a gradient similar to that found for the A 521 relic.

\section{Integrated radio spectrum of the relic}

The wealth of multifrequency radio observations available for the relic in A 521 enables its integrated spectrum to be determined over almost two orders of magnitude in frequency.

In Table 4, we report all available flux densities of the relic, along with the resolution of the images used for the measurement ${ }^{3}$. To obtain a consistent measurement of the total flux densities, we integrated over the same region in all images, and subtracted the flux density of the embedded point-sources, as measured in the corresponding full resolution images. The source subtraction could not be applied to the $74 \mathrm{MHz}$ data, since the low resolution $\left(80^{\prime \prime} \times 80^{\prime \prime}\right)$ of the Very Low-frequency Sky Survey $\left(\mathrm{VLSS}^{4}\right)$ image did not allow such an operation. Furthermore, the extended structure visible in the VLSS image is not fully consistent with the relic morphology at higher frequencies; this is probably due to the different angular resolution and much lower sensitivity of the VLSS image (which has an average rms noise of approximately $\left.1 \sigma=0.1 \mathrm{Jy} \mathrm{b}^{-1}\right)$. For these reasons, the flux density measurement at $74 \mathrm{MHz}$ is very uncertain.

The integrated spectrum of the relic is shown in Fig. 8. The spectrum does not show any steepening up to $4.9 \mathrm{GHz}$ and is well fitted by a single power law with slope $\alpha=1.48 \pm 0.01$ (solid line) between $235 \mathrm{MHz}$ and $4.9 \mathrm{GHz}$. Given its large uncertainty, the $74 \mathrm{MHz}$ data point was not included in the fit. We note that the $4.9 \mathrm{GHz}$ flux density should be considered a lower limit,

\footnotetext{
3 The table provides also the source flux density at $235 \mathrm{MHz}$ measured on a preliminary image from GMRT observations (see Appendix).

${ }^{4}$ http://lwa.nrl.navy.mil/VLSS/
} 

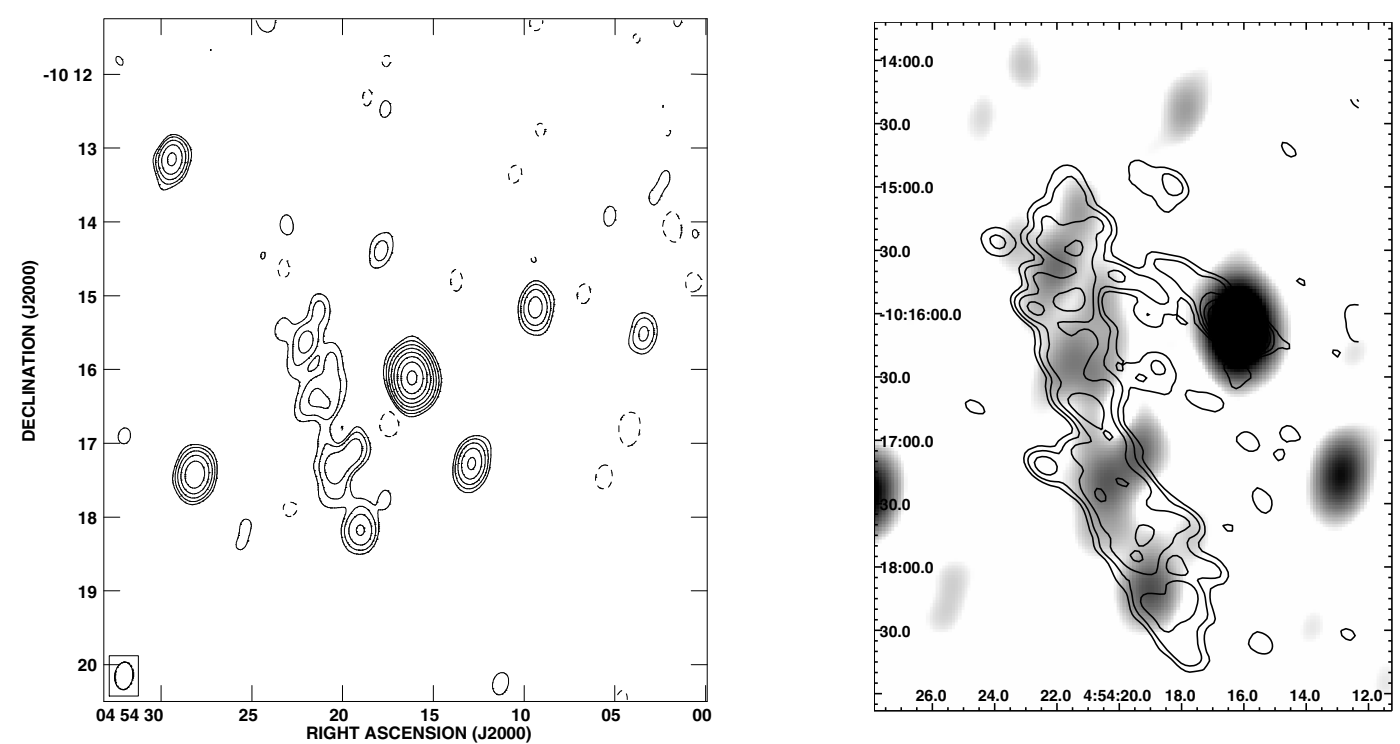

Fig. 5. VLA 4.9 GHz image of the radio relic as contours (left panel), and grey scale on the GMRT $610 \mathrm{MHz}$ image (right panel). The resolution of the $4.9 \mathrm{GHz}$ image is $22.0^{\prime \prime} \times 15.0^{\prime \prime}$, and the $1 \sigma$ noise level is $15 \mu \mathrm{Jy} \mathrm{b}^{-1}$. The resolution of the $610 \mathrm{MHz}$ image is $13.1^{\prime \prime} \times 8.1^{\prime \prime}$, and the $1 \sigma$ noise level is $40 \mu \mathrm{Jy} \mathrm{b}^{-1}$. The radio contours are spaced by a factor 2 , starting from $\pm 0.045 \mathrm{mJy} \mathrm{b}^{-1}$ and $\pm 0.012 \mathrm{mJy} \mathrm{b}^{-1}$ at $4.9 \mathrm{GHz}$ (left panel) and $610 \mathrm{MHz}$ (right panel), respectively.
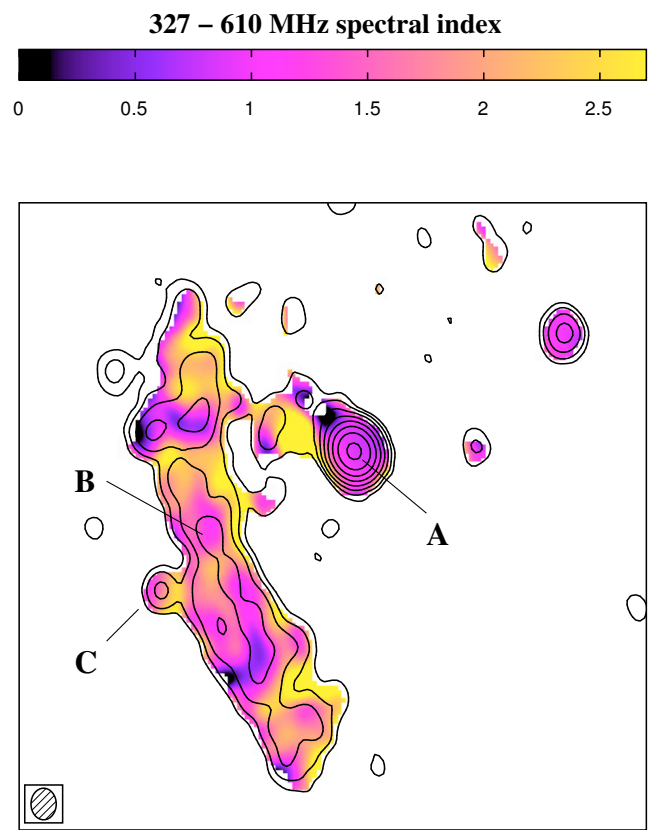

Fig. 6. Spectral image of the relic in A 521 in the 327-610 MHz range, with the GMRT $610 \mathrm{MHz}$ contours at levels $0.12,0.24,0.48,0.96,2$, $4 \mathrm{mJy} \mathrm{b}^{-1}$ overlaid. In both images (spectral index and total intensity) the resolution is $15^{\prime \prime} \times 12^{\prime \prime}$, PA $0^{\circ}$, as shown by the ellipse in the upperleft corner of the image. $\mathrm{A}, \mathrm{B}$ and $\mathrm{C}$ indicate the position of radio galaxies embedded in the relic emission (see Figs. 2 and 3).

given the array, $u-v$ coverage, and resolution which led to its detection (see Sect. 4.2).

New deep observations carried out with the VLA at 74 and $327 \mathrm{MHz}$ (October 2007 and February 2008) will allow us to better constrain the low frequency end of the radio spectrum.

\section{Discussion}

A preliminary discussion of the formation of the radio relic in A 521 was carried out in GVB06, where a number of possible theoretical frameworks were taken into account, all related to the assessed ongoing merging activity in this cluster. Two possible scenarios were considered, both invoking a tight connection with the presence of a merger-driven shock front at the location of the relic. Such a shock may accelerate electrons to ultrarelativistic energies (Enßlin et al. 1998; Roettiger et al. 1999; Hoeft \& Brüggen 2007), or it may revive fossil radio plasma through adiabatic compression of the magnetic field (Enßlin \& Gopal-Krishna 2001).

A third alternative scenario was also proposed, based on the hypothesis of a physical link between the diffuse emission of the relic and the nearby radio galaxy J0454-1016a, as suggested by the faint radio bridge of emission between the two sources observed at $610 \mathrm{MHz}$ (e.g., Fig. 5, right panel). The high frequency images of J0454-1016a (Fig. 3) and the spectral analysis of the radio relic presented in this paper (Sects. 5 and 6 ) allow us to carry out a more detailed comparison between the expectations of the models and the observed properties of the relic.

\subsection{Connection of J0454-1016a with the radio relic}

GVB06 proposed that the relic might be the result of the ram pressure stripping of the radio lobes of J0454-1016a by either $i$ ) group merging activity in the southern cluster region; or ii) the infall of the radio galaxy itself into the cluster. On the basis of pressure arguments, and given the projected distance of J0454-1016a from the relic, they estimated that the infall velocity of the merging group, or of the galaxy itself, should be $\gtrsim 3000 \mathrm{~km} \mathrm{~s}^{-1}$ (leading to a shock with Mach number $M \gtrsim 2$ ) to allow the electrons in the radio lobes to still emit in the radio band.

The high resolution and high frequency observations presented in Sect. 4.1 do not appear to support this scenario, since there is no obvious morphological link between the relic and J0454-1016a. The radio galaxy has a head-tail morphology, 

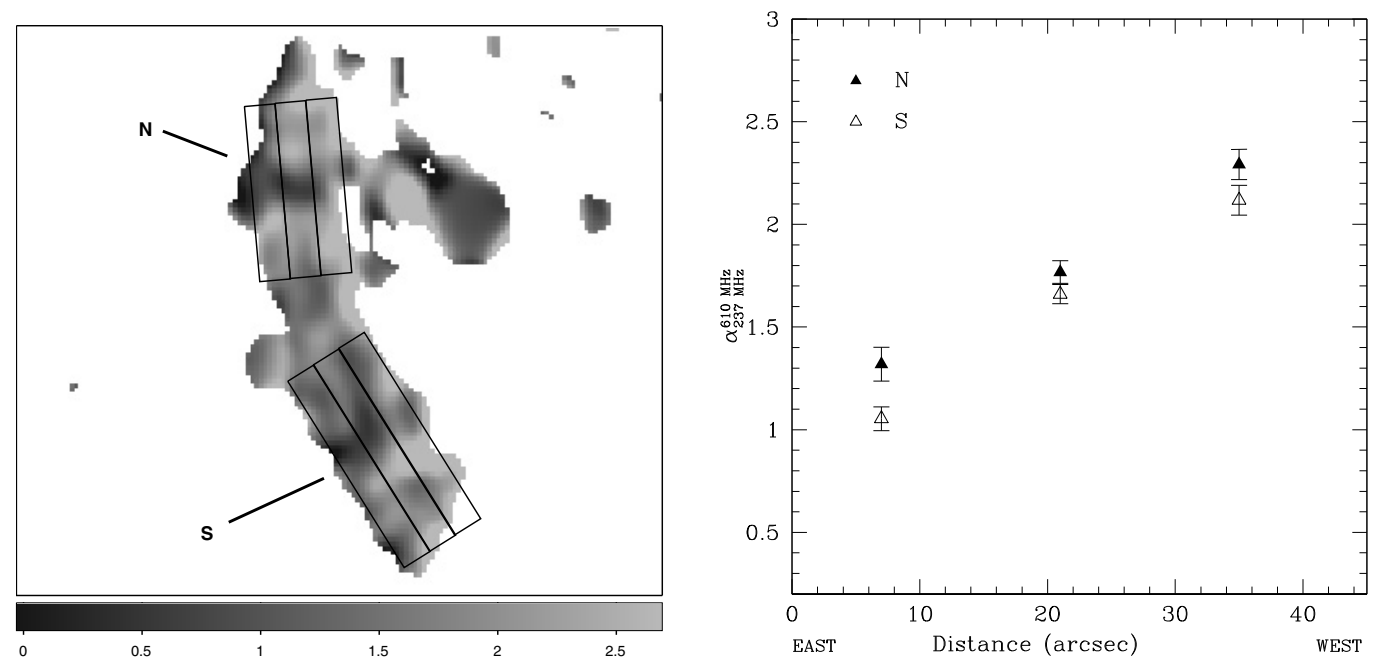

Fig. 7. Left - Grids used to derive the spectral index trend overlaid on the 327-610 MHz spectral index image (grey scale; same as Fig. 6). Right Trend of the spectral index $\alpha_{327}^{610}$ in the northern (filled triangles) and southern (empty triangles) parts of the relic.

Table 4. Flux density values of the relic.

\begin{tabular}{lccc}
\hline \hline $\begin{array}{l}v \\
(\mathrm{MHz})\end{array}$ & $\begin{array}{c}S_{v} \\
(\mathrm{mJy})\end{array}$ & $\begin{array}{c}H P W B \\
" \times{ }^{\prime \prime}\end{array}$ & Ref. \\
\hline 74 & 660 & $80.0 \times 80.0$ & VLSS \\
235 & $180 \pm 10$ & $15.6 \times 12.4$ & see Appendix \\
327 & $114 \pm 6$ & $16.0 \times 13.0$ & this work; Fig. 2 \\
610 & $42 \pm 2$ & $13.0 \times 8.1$ & GVB06 \\
1410 & $14 \pm 1$ & $15.0 \times 12.0$ & Giacintucci (2007) ${ }^{\star}$ \\
4890 & $2.0 \pm 0.2$ & $22.0 \times 15.0$ & this work; Fig. 5 \\
\hline
\end{tabular}

$\star$ From VLA-CnB archival data (Obs. Id. AF 0390).

whose tails extend (in projection) in a direction that is almost opposite to that towards the relic region and the faint bridge of emission connecting the galaxy and the relic (Fig. 3). Even though the origin of the faint bridge remains unclear, it appears to be unrelated to the current AGN activity of J0454-1016a.

\subsection{Basic expectations from the shock scenarios and comparison with the observations}

The shock scenarios for the origin of radio relics provide a number of expectations, which can be tested by means of comparison with the observed properties of the source.

a) Shock acceleration - the relic emission is produced by electrons accelerated from the thermal gas to relativistic energies by the passage of a merger shock wave (Enßlin et al. 1998; Roettiger et al. 1999; Hoeft \& Brüggen 2007). Assuming linear shock acceleration theory, in the case of a fully ionised plasma as the ICM, the steady-state energy spectrum of the electrons in the relic is a power law whose slope $\delta$ is related to the shock Mach number $M$ according to the following equation (e.g., Blandford \& Eichler 1987):

$\delta=2 \frac{M^{2}+1}{M^{2}-1}+1$

where the effect of particle aging $\Delta(\delta)=1$, from the combined effect of inverse Compton energy losses and continuous injection, is included (Sarazin 1999). The ensuing integrated radio emission of the relic has a single power law spectrum

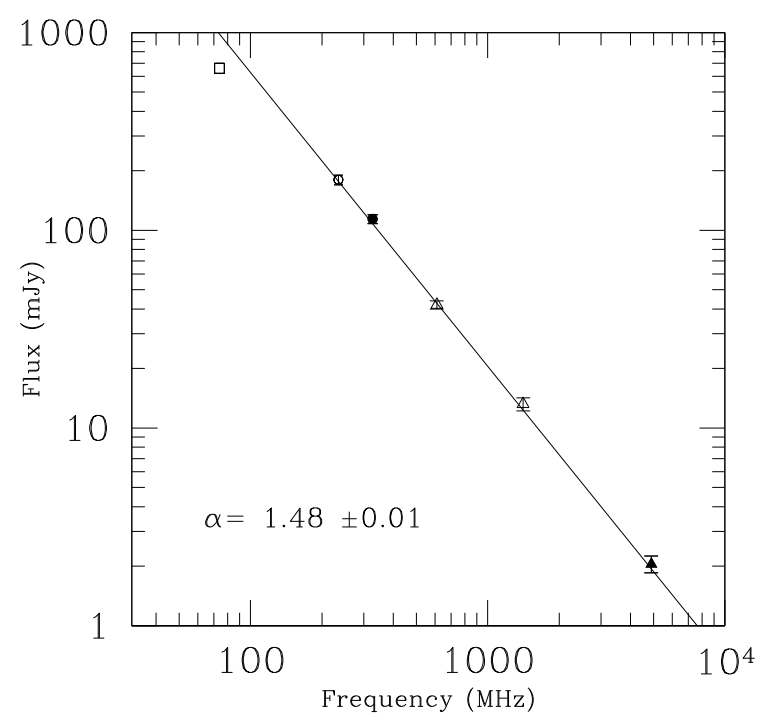

Fig. 8. Radio spectrum of the radio relic between $74 \mathrm{MHz}$ and $4.9 \mathrm{GHz}$. The solid circle is the relic flux density measured on the new GMRT image at $327 \mathrm{MHz}$. The empty triangles represent the $610 \mathrm{MHz}$ data point from GVB06, and the $1.4 \mathrm{GHz}$ value from VLA archival data. The solid triangle is the source flux density measured on the new VLA image at $4.9 \mathrm{GHz}$. The empty circle is the flux density from a preliminary GMRT image at $235 \mathrm{MHz}$ (see Appendix). The empty square is the $74 \mathrm{MHz}$ flux density from the VLSS image. The solid line represents the linear fit to the data by using the points between $235 \mathrm{MHz}$ and $4.9 \mathrm{GHz}$.

with $\alpha=(\delta-1) / 2$, which is thus related to the Mach number of the shock through Eq. (1). More specifically, the total spectrum results from the combination of the aged spectra of the injected electrons: once accelerated, the electrons are short-lived because of the inverse Compton and synchrotron energy losses, and their spectrum rapidly steepens with the distance from the shock rim. Thus, the spectral index distribution of the relic synchrotron radio emission should exhibit a progressive steepening going from the current location of the shock (where the observed $\alpha$ is the injection spectral index) to the trailing edge.

b) Adiabatic compression - the relic is the result of the adiabatic compression exerted by a merger shock on a region 
containing fossil radio plasma (Enßlin \& Gopal-Krishna 2001). Such compression might increase the magnetic field strength and re-energize the electron population in the fossil plasma, thus leading to observable radio emission. In this case, the relativistic electrons are expected to produce diffuse radio emission in front of the bow shock, and then rapidly lose their energy while moving away from the front. Hence, a transversal steepening in the spectral index distribution of the relic may be expected in this model. Unlike the shock acceleration case, this scenario predicts a curved integrated radio spectrum showing a spectral steepening at high frequency, at least for relatively weak shocks $(M \lesssim 3)$.

c) Shock re-acceleration - This scenario was discussed by Markevitch et al. (2005) for the origin of the radio edge coincident with the shock front in A 520. The relic is proposed to be produced by the re-acceleration of fossil electrons in the ICM by the shock. The basic expectations for the spectral properties of the relic are similar to the shock acceleration scenario $(a)$.

The results of the spectral analysis presented in this paper provide insightful information to discriminate between the models described above. In the case of the relic in A 521, a significant steepening of the spectral index was found going from the eastern edge of the source toward the western border (Fig. 7). This behaviour is in line with the expectations of all the scenarios. It implies that the shock is expected to be moving outwards with respect to the cluster centre, and its current location should be approximately coincident with the eastern edge of the relic. In Sect. 6, we showed that the integrated spectrum of the relic is well reproduced by a single power law with steep spectral index $(\alpha \sim 1.5)$, and with no evidence of high frequency steepening up to $4.9 \mathrm{GHz}$ (Fig. 8). Such a spectral shape is expected only in the framework of the shock acceleration and re-acceleration scenario.

The adiabatic compression scenario should produce curved spectra with a high frequency cut-off in the case of moderate or weak shocks, such as those expected to be developed during cluster mergers (e.g., Gabici \& Blasi 2003; Ryu et al. 2003; Pfrommer et al. 2006, and references therein). In principle, a cut-off just above $5 \mathrm{GHz}$ cannot be ruled out. The compression enhances the magnetic field and the particle energy density, moving the break frequency in the synchrotron spectrum to higher frequencies. Assuming thermal and relativistic particles mixed into the fossil plasma, the ratio of the post-compression and initial break frequencies ( $v_{\mathrm{b}}^{\text {post }}$ and $v_{\mathrm{b}}^{0}$, respectively) is (e.g., Markevitch et al. 2005):

$\frac{v_{\mathrm{b}}^{\text {post }}}{v_{\mathrm{b}}^{0}}=\left(\frac{4 M^{2}}{M^{2}+3}\right)^{4 / 3}<6$.

This value is not sufficiently high to explain the lack of a cut-off in the observed spectrum of the relic ${ }^{5}$.

This factor can be strongly increased if the shock compression acts on a ghost of purely relativistic plasma (i.e., not mixed with the thermal ICM). In this case (Enßlin \& Gopal-Krishna 2001):

$\frac{\nu_{\mathrm{b}}^{\mathrm{post}}}{v_{\mathrm{b}}^{0}} \approx \frac{P_{2}}{P_{1}} \approx \frac{5 M^{2}-1}{4}$

where $P_{1}$ and $P_{2}$ are the pre- and post-shock thermal pressures. To produce a spectral break at $v \geq 5 \mathrm{GHz}$, a shock with $M \geq 7$ is

\footnotetext{
5 Adopting a viable value of $v_{\mathrm{b}}^{0}=50-100 \mathrm{MHz}$ in a fossil radio plasma.
}

required, which however would imply a very unlikely pre-shock temperature of $T<1 \mathrm{keV}$ for A 521 .

An additional point is that the injection spectrum of the fossil radio plasma in this scenario should be roughly equal to the observed one ( $\alpha \sim 1.5)$, which is much steeper than the typical spectra of radio galaxies.

To summarise, our spectral analysis suggests that the origin of the relic in A 521 is consistent with the shock acceleration scenario. According to Eq. (1), we can estimate the Mach number of the shock responsible for the electron acceleration. The spectral fit provided a total spectral index $\alpha=1.48 \pm 0.01$ (Sect. 6), which corresponds to $\delta=3.96 \pm 0.02$, and thus $M(\delta)=2.27 \mp 0.02$. Such a Mach number is in reasonable agreement with the values expected for the cluster merger shocks, and indeed observed in merging clusters (e.g., Markevitch \& Vikhlinin 2007). As argued in GVB06, the relic is located in a peripheral region of A 521, which is expected to be dynamically active (Maurogordato et al. 2000; Ferrari et al. 2003, 2006; see also Fig. 2 in GVB06). Thus the presence of a shock front at the relic location is likely.

\subsection{A shock front coincident with the radio relic?}

Using the available Chandra X-ray archival data of A 521, we checked if there is indeed a shock front in the cluster gas at the position of the relic. Shock fronts in clusters are observed very rarely. Only two unambigous examples, exhibiting both a sharp gas density edge and a clear gas temperature jump, have been discovered by Chandra so far, those in the merging clusters 1E 0657-56 (Markevitch et al. 2002) and A 520 (Markevitch et al. 2005). Their rarity is due to the fact that the viewing geometry and the moment of our observation must be favourable: a merger shock quickly moves to the cluster outskirts where it cannot be detected.

For A 521, two observations are available in the Chandra public archive, one performed using ACIS-I and another using ACIS-S, with $\sim 40 \mathrm{ks}$ exposure each (Ferrari et al. 2006). Unfortunately, the relic lies right at the S3 chip boundary in the ACIS-S observation, so we could use only the ACIS-I observation (OBSID 901) for our purpose. We cleaned the data and modelled the detector background and instrumental spatial response as described in Vikhlinin et al. (2005).

In Fig. 9, we show the resulting Chandra image in the $0.5-4.0 \mathrm{keV}$ energy range. The background was subtracted and the image divided by the exposure map, and then smoothed with a $\sigma=6^{\prime \prime}$ Gaussian. In the right panel, the GMRT $610 \mathrm{MHz}$ contours are overlaid on the same X-ray image. The image reveals a clear brightness edge coincident with the outer edge of the radio relic.

The radial brightness profile in Fig. 10 (left panel) shows this edge more clearly. The profile was extracted from the unsmoothed image in the sector shown in the left panel of Fig. 9, which is centred on the centre of curvature of the relic and spans the angle covered by the source. Discrete X-ray sources were excluded for the profile derivation. Figure 10 (left panel) shows a brightness edge at $r=300^{\prime \prime}$, which coincides with the outer edge of the radio relic; we note that the drop in the profile and increased error bar at $r \approx 200^{\prime \prime}$ corresponds to an interchip gap. For comparison, in the right panel we plot the X-ray brightness profiles extracted from three other sectors of the cluster. None of these profiles show such an edge, which is consistent with the visual inspection of the image shown in Fig. 9.

The X-ray edge in Fig. 10 (left panel) has the characteristic shape that corresponds to a projection of a spherical density discontinuity. To quantify the discontinuity, we fitted the brightness 

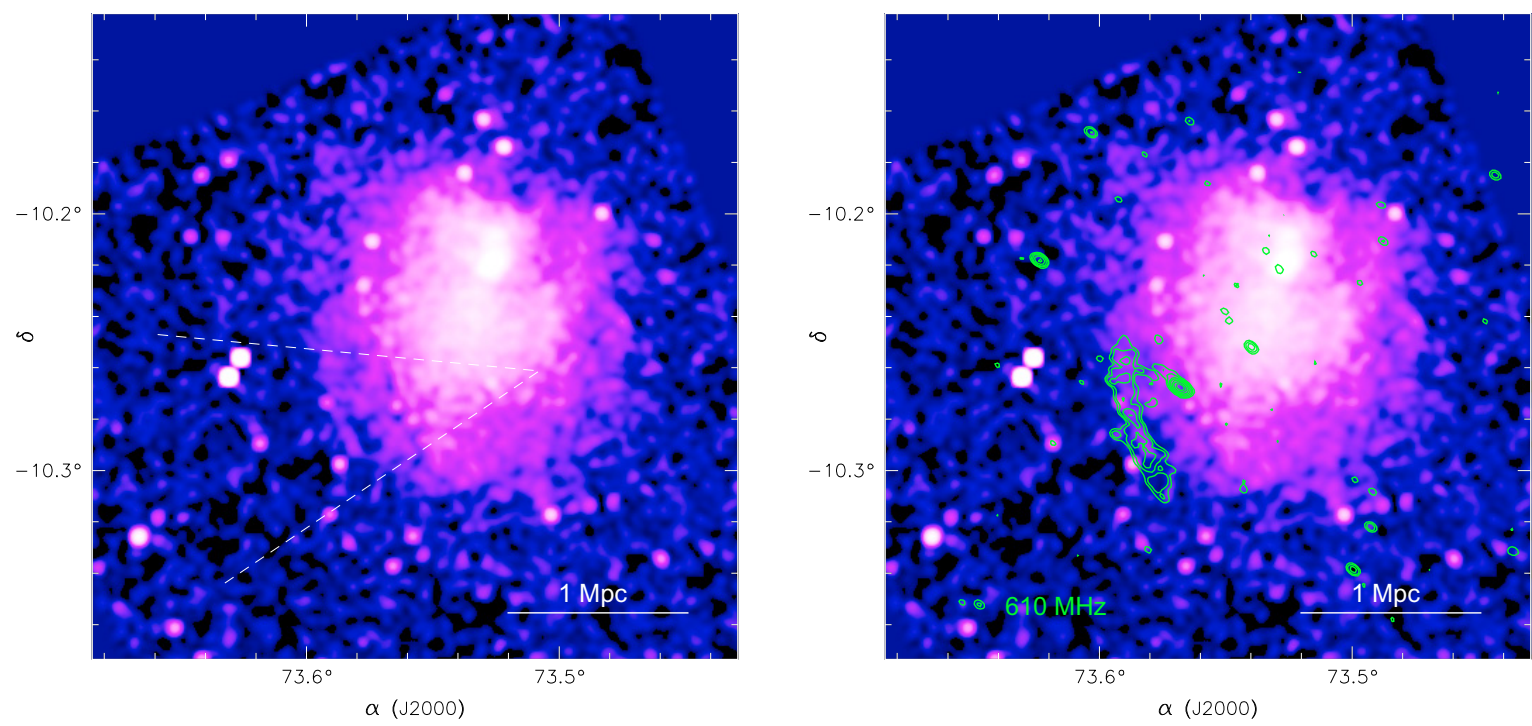

Fig. 9. Left panel - Smoothed Chandra image of A 521 in the $0.5-4 \mathrm{keV}$ energy band. Right panel - Same image as left panel with the GMRT $610 \mathrm{MHz}$ contours of the A 521 field overlaid. The resolution of the radio image is $13.1^{\prime \prime} \times 8.1^{\prime \prime}$, PA $56^{\circ}$. Contour levels are spaced by a factor 2 starting from $0.2 \mathrm{mJy} \mathrm{b}^{-1}$.
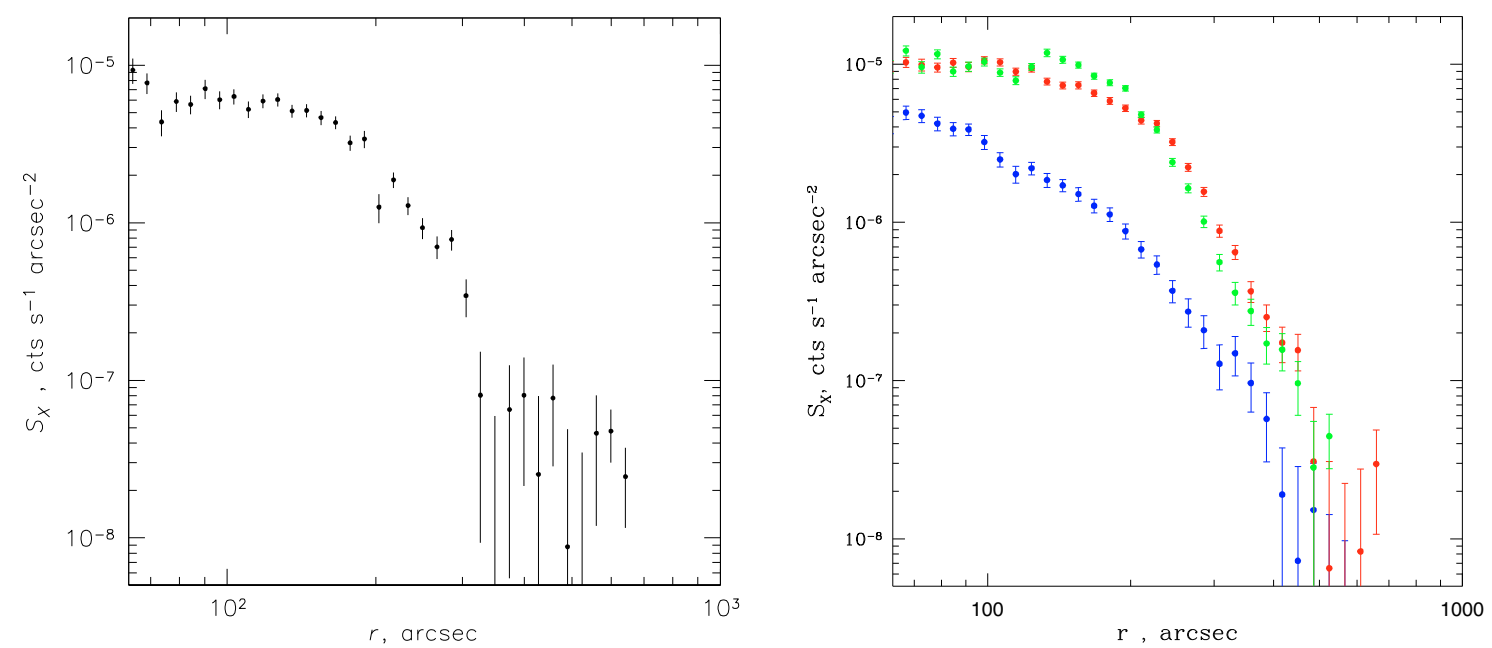

Fig. 10. Left panel: X-ray $0.5-4.0 \mathrm{keV}$ brighntess profile across the edge in the relic region, extracted in the sector indicated in the left panel of Fig. 9. Error bars are $1 \sigma$. Right panel: X-ray $0.5-4.0 \mathrm{keV}$ brightness profiles extracted in sectors covering the South-West (blue), North-East (red) and North-West (green) portions of the cluster. Error bars are $1 \sigma$.

profile close to the edge using a gas-density model of two power laws and an abrupt jump, where the power laws and position and amplitude of the jump were free parameters (as in Markevitch et al. 2000). A continuous density profile (i.e., no jump, but a possible break), shown by the red line in Fig. 11, is inconsistent with the data at about $4.5 \sigma$. If one assumes a $6 \mathrm{keV}$ gas temperature just inside the edge (Ferrari et al. 2006, determined from this and the ACIS-S Chandra observations) and converts brightness in the Chandra $0.5-4 \mathrm{keV}$ band into plasma emission measure self-consistently, the best-fit density jump (the model shown by green line) corresponds to $M \approx 7$. However, as mentioned above, such a shock would imply a pre-shock temperature of $\sim 0.4 \mathrm{keV}$, which is unlikely close to the centre of such a hot cluster. A shock with $M=2.3$, predicted from the radio spectrum (Sect. 7.2), corresponds to an edge shown by the blue line in Fig. 11; this fit is only $1.8 \sigma$ away from the best fit, which we consider good agreement.

Unfortunately, the accuracy of the existing Chandra observation is insufficient to measure the gas temperature on the faint side of the edge. Thus, we cannot rule out other interpretations of this feature, e.g., a cold front (Markevitch \& Vikhlinin 2007). However, a cold front with such a density contrast would correspond to an outer gas temperature in excess of $15 \mathrm{keV}$ - quite unlikely in a cluster of this X-ray luminosity. We conclude that the X-ray edge is most likely a shock front - found at a location and with an amplitude exactly as required to produce the radio relic by means of the shock acceleration mechanism.

\section{Summary and conclusions}

We presented new deep radio images of the radio relic in A 521 obtained using the GMRT at $327 \mathrm{MHz}$ and the VLA at 4.9 and 8.5 GHz. We performed a study of the spectral properties of the source by combining the new radio information with our previous GMRT data at $610 \mathrm{MHz}$ (GVB06) and $1.4 \mathrm{GHz}$ observations available in the VLA public archive and reanalysed in Giacintucci (2007). 


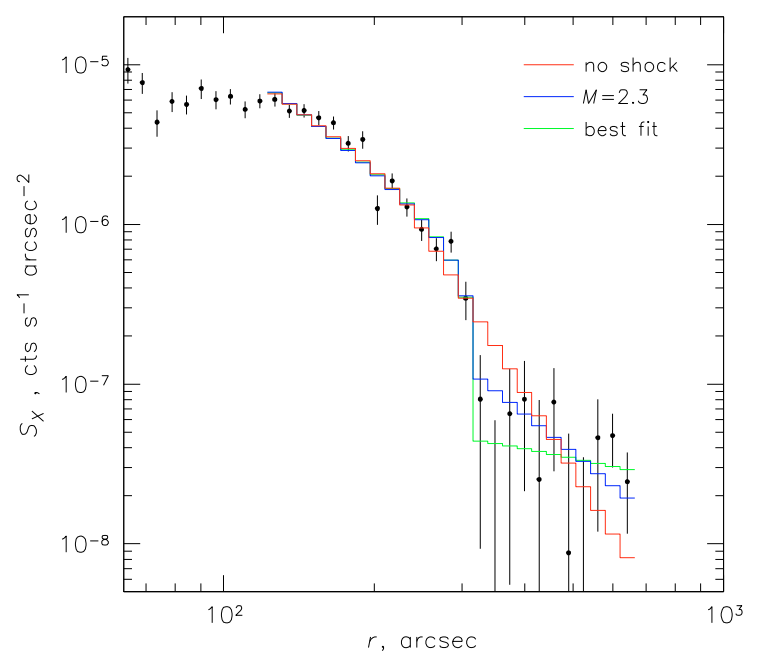

Fig. 11. X-ray brighntess profile (same as left panel of Fig. 10) compared to different models. The green and red lines are the fit with and without a shock discontinuity, respectively. The blue line is the profile expected for a shock with $M=2.3$.

In the following, we summarise the main results of our analysis:

1. The relic morphology at $327 \mathrm{MHz}$ is in overall agreement with the structure observed at $610 \mathrm{MHz}$ and $1.4 \mathrm{GHz}$.

2. The relic was detected at $4.9 \mathrm{GHz}$ at a high level of significance (from $3 \sigma$ in the fainter regions to $12 \sigma$ at the peaks). This is the second detection of a radio relic at a frequency as high as $4.9 \mathrm{GHz}$ after the relic source $1253+275$ in the Coma cluster (Thierbach et al. 2003).

3. A faint bridge of emission is clearly detected between the northern part of the relic and the cluster radio galaxy J0454-1016a. This emission was also observed at $610 \mathrm{MHz}$, and suggested the existence of a physical connection between the two sources. The high resolution images at 4.9 and 8.5 GHz appear to rule out this hypothesis: J0454-1016a has a head-tail structure that extends in a direction opposite to that of the bridge, whose origin remains thus unclear.

4. The spectral index image of the relic between 327 and $610 \mathrm{MHz}$ looks rather patchy and irregular. However, our analysis revealed a significant gradual steepening of the spectral index along the source minor axis, from the outer edge of the relic toward the cluster centre. A similar trend has been observed in a few other relics with available spectral index images, as for example the case of A 2256 where a spectral study similar to the analysis presented in this paper was performed by Clarke \& Enßlin (2006).

5. We derived the integrated spectrum of the relic over almost two orders of magnitude in frequency. Such a frequency coverage is available for a few relics only (e.g., the Coma cluster relic; Thierbach et al. 2003). The spectrum of the relic in A 521 is well fitted by a power law with a steep spectral index ( $\alpha \sim 1.5$ ), with no evidence of a steepening in the high frequency regime (up to $4.9 \mathrm{GHz}$ ). This situation is similar to the Coma relic, which shows a single power law spectrum with $\alpha \sim 1.2$.

6. We analysed the archival Chandra observation of A 521 and discovered a clear X-ray brightness edge at the position of the outer border of the radio relic. This edge can be the shock front responsible for the electron acceleration. The present $\mathrm{X}$-ray data accuracy is insufficient to confirm that it is a shock front, but the coincidence is tantalizing.

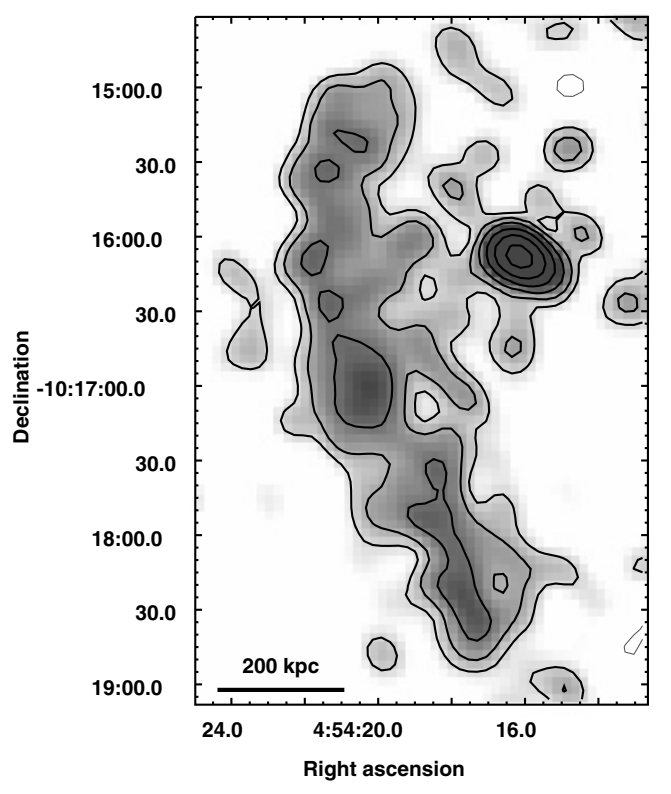

Fig. A.1. Preliminary GMRT image (contours and grey scale) of radio relic at $235 \mathrm{MHz}$. The resolution is $15.6^{\prime \prime} \times 12.4^{\prime \prime}$, in PA 56. The noise level is $1 \sigma=270 \mu \mathrm{J} \mathrm{b}^{-1}$. Contours are spaced by a factor 2 starting from $\pm 3 \sigma$.

The results of our spectral analysis were discussed in the framework of the possible models for the relic origin considered in GVB06. We concluded that the most plausible scenario is shock acceleration, while adiabatic compression appears to be ruled out by the present data. In the shock acceleration case, the flattest spectrum emission of the relic (i.e., the outer region) would mark the position of the shock front, where the electron acceleration is expected to be currently ongoing. This suggests that the shock wave is travelling from the northwestern direction towards the South-East, as projected on the plane of the sky. The possible merging scenario for A 521 (Ferrari et al. 2003, 2006) might be consistent with such hypothesis. The presence of an edge in the X-ray surface brightness in the relic region suggests the existence of a shock front which may be responsible for the electron acceleration in the relic. Deeper X-ray observations are required to confirm the presence of this shock.

Acknowledgements. We thank the staff of the GMRT for their help during the observations. GMRT is run by the National Centre for Radio Astrophysics of the Tata Institute of Fundamental Research. Thanks are due to E. Fomalont for his help with the use of the NRAO pipeline. We thank the anonymous referee for useful comments. S.G. and T.V. acknowledge partial support from the Italian Ministry of Foreign Affairs. This work has been partially supported by contracts ASI-INAF I/088/06/0, PRIN-MUR 2005, and PRIN-INAF 2005.

\section{Appendix A: Preliminary results of the GMRT follow up at $235 \mathrm{MHz}$}

In this Appendix, we present the preliminary $235 \mathrm{MHz}$ image of the relic obtained from recent GMRT data. The source was observed on 2007, November 11th and 13rd, for a total integration time of $\sim 18 \mathrm{~h}$. The observations were carried out in spectral line mode using only the USB for a total bandwidth of $8 \mathrm{MHz}$ (128 channels, spectral resolution $62.5 \mathrm{kHz} / \mathrm{channel}$ ). The data reduction was performed as described in Sect. 2.1.

The full resolution image shown in Fig. A.1 was produced using only the data from the first day (i.e., $\sim 9$ h). The image has a very high sensitivity level $\left(1 \sigma=270 \mu \mathrm{Jy} \mathrm{b}^{-1}\right)$, and the relic is clearly detected and imaged in its whole extent. Given 
the high quality of this image we are confident that the flux density given in Table 4 is definitely reliable, with an error of the order of $5 \%$. The analysis of the entire dataset (i.e., from the combination of the two observing days) will be presented in a forthcoming paper.

\section{References}

Andernach, H., Feretti, L., \& Giovannini, G. 1984, A\&A, 133, 252 Arnaud, M., Maurogordato, S., Slezak, E., \& Rho, J. 2000, A\&A, 355, 461 Blandford, R., \& Eichler, D. 1987, Phys. Rep., 154, 1

Brunetti, G. 2003, in Matter and Energy in Clusters of Galaxies, ed. S. Bowyer, \& C.-Y. Hwang (San Francisco: Astron. Soc. Pac.), ASP Conf. Ser., 301, 349 Brunetti, G. 2004, in Outskirts of Galaxy Clusters: Intense life in the suburbs, ed. A. Diaferio, IAU Coll., 195, 148

Brunetti, G., Venturi, T., Dallacasa, D., et al. 2007, ApJ, 670, L5

Cassano, R., \& Brunetti, G. 2005, MNRAS, 357, 1313

Cassano, R., Brunetti, G., \& Setti, G. 2006, MNRAS, 369, 1577

Cassano, R., Brunetti, G., Venturi, T., et al. 2008, A\&A, 480, 687

Clarke, T. E., \& Enßlin, T. A. 2006, AJ, 131, 2900

Condon, J. J., Cotton, W. D., Greisen, E. W., et al. 1998, AJ, 115, 1693

Enßlin, T. A., \& Gopal-Krishna, 2001, A\&A, 366, 26

Enßlin, T. A., Biermann, P. L., Klein, U., et al. 1998, A\&A, 332, 395

Feretti, L. 2005, in X-Ray and Radio Connections, ed. L. O. Sjouwerman, \& K. K. Dyer, Published electronically by NRAO at http://www . aoc . nrao. edu/events/xraydio

Ferrari, C., Maurogordato, S., Cappi, A., et al. 2003, A\&A, 399, 813

Ferrari, C., Arnaud, M., Ettori, S., et al. 2006, A\&A, 446, 417
Gabici, S., \& Blasi, P. 2003, ApJ, 583, 695

Giacintucci, S. 2007, Ph.D. Thesis, University of Bologna

Giacintucci, S., Venturi, T., Bardelli, S., et al. 2006, NewA, 11, 437 (GVB06)

Giovannini, G., Feretti, L., \& Stanghellini, C. 1991, A\&A, 252, 528

Hoeft, M., \& Brüggen, M. 2007, MNRAS, 375, 77

Kardashev, N. S. 1962, SvA, 6, 317

Kuo, P.-H., Hwang, C.-Y., \& Ip, W.-H. 2004, ApJ, 604, 108

Markevitch, M., \& Vikhlinin, A. 2007, PhR, 443, 1

Markevitch, M., Ponman, T. J., Nulsen, P. E. J., et al. 2000, ApJ, 541, 542

Markevitch, M., Gonzalez, A. H., David, L., et al. 2002, ApJ, 567, 27

Markevitch, M., Govoni, F., Brunetti, G., et al. 2005, ApJ, 627, 733

Maurogordato, S., Proust, D., Beers, T. C., et al. 2000, A\&A, 355848

Murgia, M. 2001, Ph.D. Thesis, University of Bologna

Orrú, M., Murgia, M., Feretti, L., et al. 2007, A\&A, 467, 9430

Parma, P., Murgia, M., de Ruiter, H. R., et al. 2002, NewAR, 46, 313

Petrosian, V., \& Bykov, A. 2008 in Clusters of galaxies: beyond the thermal view, ed. J. S. Kaastra [arXiv:0801.0923]

Pfrommer, C., Springel, V., Enßlin, T. A., \& Jubelgas, M. 2006, MNRAS, 367, 113

Roettiger, K., Burns, J. O., \& Stone, J. M. 1999, ApJ, 518, 594

Röttgering, H. J. A., Wieringa, M. H., Hunstead, R. W., \& Ekers, R. D. 1997, MNRAS, 290, 577

Ryu, D., Kang, H., Hallman, E., \& Jones, T. W. 2003, ApJ, 593, 599

Sarazin, C. L. 1999, ApJ, 520, 529

Sarazin, C. L. 2004, JKAS, 37, 433

Thierbach, M., Klein, U., \& Wielebinski, R. 2003, A\&A, 397, 53

Venturi, T., Giacintucci, S., Brunetti, G., et al. 2007, A\&A, 463, 937

Venturi, T., Giacintucci, S. D., Dallacasa, et al. 2008, A\&A, 484, 327

Vikhlinin, A., Markevitch, M., Murray, S. S., et al. 2005, ApJ, 628, 655 